\title{
Inflammatory Response in Preterm Infants Is Induced Early in Life by Oxygen and Modulated by Total Parenteral Nutrition
}

\author{
PASCAL M. LAVOIE, JEAN-CLAUDE LAVOIE, CARLA WATSON, THÉRÈSE ROULEAU, BRENT A. CHANG, \\ AND PHILIPPE CHESSEX
}

\author{
Division of Neonatology [P.M.L., C.W., B.A.C., P.C.], Children's and Women's Health Centre of British Columbia, University of British \\ Columbia, Vancouver, British Columbia, Canada V6H 3V4; Department of Pediatrics [J.C.L., T.R.], Hospital Ste-Justine, University of \\ Montreal, Montreal, Quebec, Canada H3T 1 C5
}

\begin{abstract}
The i.v. lipid emulsion (LIP) is a source of oxidants, which may stimulate inflammation. Coadministration of parenteral multivitamins (MVP) with LIP prevents lipid peroxidation in lightexposed total parenteral nutrition (TPN). We hypothesized that this modality of TPN administration affects systemic inflammation, which may be modulated by exposure to oxygen. Premature infants were allocated to three TPN regimens: control regimen - MVP coadministered with amino acid/dextrose exposed to ambient light, LIP provided separately $(n=9)-$ LIP + MVP light exposed (LE): MVP coadministered with light-exposed LIP $(n=9)-$ LIP+MVP light protected (LP): MVP coadministered with light-protected LIP $(n=8)$. In LE and LP, amino acid/dextrose was provided separately. On reaching full TPN, infants were sampled for IL- 6 and IL- 8 in plasma and the redox potential of glutathione in whole blood $(E, \mathrm{mV})$. Data were compared (ANOVA) in infants exposed to low $(<0.25)$ versus high $(\geq 0.25) \mathrm{FiO}_{2}$. Patients (mean $\pm \mathrm{SD}$ : birth weight $797 \pm$ $172 \mathrm{~g}$; GA $26 \pm 1 \mathrm{wk}$ ) had similar clinical characteristics in TPN groups. Cytokine levels correlated positively $(p<0.01)$ with $\mathrm{FiO}_{2}$ and $E$. High $\mathrm{FiO}_{2}$ stimulated an increase $(p<0.01)$ in cytokines in control regimen, whereas these markers remained unaffected by oxygen in the LE and LP groups. The choice of a TPN admixture may have important consequences on the systemic inflammatory response triggered by an oxidant stress. (Pediatr Res 68: 248-251, 2010)
\end{abstract}

$\mathrm{S}$ upplemental oxygen, assisted ventilation, and total parenteral nutrition (TPN) are therapies that expose premature infants to significant oxidant and inflammatory stress (1-4). As these infants are particularly deficient in antioxidant and anti-inflammatory defenses, such therapies may also have potentially harmful long-term effects on their developing lungs and brain (5).

Synergistic deleterious effects of inflammation and oxidant stress in preterm infants can occur through a number of potential mechanisms. Exposure to oxygen leads to the accumulation of reactive oxygen species (ROS), which results in the generation of hydrogen and lipid peroxides. When antioxidant defenses are overwhelmed, this reaction self-propagates, and the peroxides generated can damage cells and tissues (6). In preterm infants, even brief periods of supplemental oxygen

Received March 22, 2010; accepted May 17, 2010.

Correspondence: Philippe Chessex, M.D., Division of Neonatology, Children's and Women's Hospitals, 4480 Oak St, Vancouver, British Columbia, Canada V6H 3V4; e-mail: pchessex@hotmail.com

Supported by a Grant MOP 53270 from the Canadian Institutes of Health Research. at birth may produce sufficient oxidant stress to cause sustained lung disease (7). Also, oxidized lipids and hydrogen peroxides have a direct modulatory effect on inflammatory pathways, including Toll-like receptors (8), and the transcriptional activator nuclear factor (NF)-kB (9). Such a direct activation of inflammatory pathways by oxygen byproducts can potentiate inflammatory responses and promote further endothelial injury and pulmonary capillary leakage, resulting in systemic generalization of the inflammation with potentially harmful consequences on the developing preterm brain (10). Hydrogen peroxides are markedly increased in brain tissues of animals exposed to hyperoxia (11). Oxidant stress and systemic inflammation each have independent and synergistic deleterious apoptotic effects on brain microglial cells and immature oligodendrocyte (12-14). Notably, prolonged supplemental oxygen exposure and systemic inflammation as measured by plasma levels of IL- 6 and IL- 8 have both been strongly associated with adverse long-term pulmonary and neurodevelopmental outcomes in preterm infants (15-19).

Quality of nutrition early in life has documented beneficial effects on health outcomes later in life (20). Premature infants often require i.v. nutritional support (TPN) until optimal gut maturity is achieved to allow full enteral feeding. Light exposure of TPN induces the loss of antioxidant vitamins (21) and the generation of oxidant products of photooxidation such as hydrogen peroxide (22), lipid peroxides (23), aldehydes (24), and ascorbylperoxide (25). The generation of these products of oxidation can be decreased by protection from light (23). It has been recently shown in vitro that multivitamin coadministration with the lipid moiety of TPN prevents lipid peroxidation and antioxidant vitamin loss in the infusate (21). Based on the previously documented antioxidant effect of light protection and coadministration of multivitamins with lipids, which limits the principal sources of peroxides in infants receiving TPN, we hypothesized that coadministration

\footnotetext{
Abbreviations: AA, control regimen; AA+MVP, parenteral multivitamin preparation mixed within the amino acid dextrose solution; $\boldsymbol{E}$, redox potential expressed in $\mathrm{mV}$; LIP+MVP, parenteral multivitamin preparation mixed within the lipid emulsion; LE, LIP+MVP light exposed; LP, LIP+MVP light protected; MVP, multivitamin preparation; NEC, necrotizing enterocolitis; ROS, reactive oxygen species; TPN, total parenteral nutrition.
} 
of i.v. multivitamins with lipids would reduce the proinflammatory effect of supplemental oxygen in the early days of life.

\section{METHODS}

Subject recruitment and intervention. Infants born at $28 \mathrm{wk}$ of gestation or lower admitted to the Children's \& Women's Health Centre of British Columbia neonatal intensive care unit between 2006 and 2009 were prospectively recruited as part of a study evaluating the antioxidant response to different TPN regimens. Written informed consent was obtained. Infants were allocated by chance in pharmacy to one of three different TPN regimens initiated in the first $24 \mathrm{~h}$ of age and continued for at least $10 \mathrm{~d}$ :

Infants in the control regimen (AA) group received parenteral multivitamin (MVP) mixed with the amino acids/dextrose solution (AA+MVP) exposed to ambient light (clear syringes and tubing), and the lipid emulsion (LIP) was administered separately.

Infants in the LIP+MVP light-exposed (LE) group received parenteral multivitamin mixed within the LIP (LIP+MVP) exposed to ambient light (clear syringes and tubing), and the amino acid/dextrose solution was provided separately.

Infants in the LIP+MVP light-protected (LP) group received parenteral multivitamin mixed within the LIP (LIP+MVP) protected from light (using amber syringes and tubing (26), and the light-exposed amino acid/dextrose solution was provided separately.

In all three groups, nutrient intake was provided according to the institutional TPN protocol (27), so that all infants in the study would have a similar nutrient intake. Inclusion criteria: premature infants with birth weight $<1000$ $\mathrm{g}$ and GA $<28$ wk who required TPN. To control for variability in the nutrient intake, subjects were excluded if enteral energy intake exceeded the parenteral energy intake at any given time during the study. To minimize blood letting, every other infant recruited in the trial was considered for plasma cytokine analyses. Two samples for plasma cytokine and whole blood redox potential analyses were collected $72 \mathrm{~h}$ apart, between days 7 and 10 of life. IL- 6 and IL-8 were specifically included in the analysis as they most strongly correlated with outcomes in previous studies (18). The study protocol was approved by the Clinical Research Ethics Board of the University of British Columbia.

Definitions. Chorioamnionitis was defined histologically as a maternal stage 2 or greater with involvement of fetal membranes, according to criteria previously established by Redline (28). The Score for Neonatal Acute Physiology (SNAP-II) was measured within $12 \mathrm{~h}$ of age (29). The fractional inspired oxygen $\left(\mathrm{FiO}_{2}\right)$ was recorded on the day of blood sampling and categorized into a low $\left(\mathrm{FiO}_{2}<0.25\right)$ or high $\left(\mathrm{FiO}_{2} \geq 0.25\right)$ exposure to oxygen. A cutoff of $25 \% \mathrm{FiO}_{2}$ was chosen to better discriminate between the clinical statuses of infants with mild versus more significant lung disease. Necrotizing enterocolitis (NEC) was defined according to the attending neonatologist if the infant presented signs of acute gastrointestinal deterioration with radiologic pneumatosis, free or portal air, or signs of fixed bowel dilatation with bowel wall thickening. Culture-proven sepsis was defined by either a positive blood or cerebrospinal fluid (CSF) culture. Because we were interested in identifying a confounding effect of NEC or sepsis on the levels of oxidant stress or inflammatory cytokines, only diagnoses occurring during the $10-\mathrm{d}$ study period were considered in the data analysis.
Laboratory analyses. Within 4 min of sampling, aliquots of whole blood (EDTA tube) were homogenized in freshly prepared metaphosphoric acid $(5 \% \mathrm{wt} / \mathrm{vol})$. The plasma fraction was separated by centrifugation within minutes of blood sampling (EDTA tube) and stored at $-80^{\circ} \mathrm{C}$ until batch analysis. Concentrations of IL-6, IL-8, IL- $1 \beta$, IL-10, IL-12, and TNF- $\alpha$ were measured in duplicate using a cytometric-bead array multiplex assay (BD Bioscience, San Diego, CA) and analyzed on a FACSCalibur flow cytometer (Becton Dickinson). Interduplicate variations were within 10\% and 6\% for the two detectable cytokines, IL-6 and IL-8, respectively. Reduced glutathione (GSH) and oxidized glutathione (GSSG) in centrifuged whole blood $(5000 \times \mathrm{g})$ were determined at $200 \mathrm{~nm}$ after separation by capillary electrophoresis (30). The redox potential of glutathione $[\mathrm{GSH} /(\mathrm{GSSG}+\mathrm{GSH}), E$, $\mathrm{mv}$, a measure of overall oxidant/antioxidant status, was calculated from the Nernst equation (31).

Statistical analysis. Cytokine data (mean $\pm \mathrm{SD}$ ) were compared by factorial ANOVA $3 \times 2 \times 2$, mode of MVP admixture $\{[\mathrm{LE}$ versus LP $($ effect of light)] versus AA $\} \times$ effect of oxygen (low versus high $) \times$ duration of TPN (sample 1 versus sample two). When indicated, data were transformed by natural log to satisfy homoscedasticity tested by Barlett's $\chi^{2}$. The orthogonality of the comparisons was reached using the harmonic mean of the sample size in each group. Correlations between oxygen exposure and cytokine levels were determined using a Spearman's rank test. Correlations between redox potential and cytokines were determined by Pearson's correlation coefficient. Statistical analyses were performed using SPSS 11.0 for windows (SPSS Inc., Chicago, IL). The level of significance was set at $p<0.05$.

\section{RESULTS}

Clinical characteristics of infants. Twenty-six preterm infants had samples drawn for determination of the redox potential and cytokines on postnatal days 7 and 10. Clinical characteristics, include anthropometric parameters and factors that contribute to generating systemic inflammation or oxidant stress, such as the presence of chorioamnionitis, blood-culture proven infections or NEC, severity of illness score (SNAP-2), are presented in Table 1. These variables, as well as nutrient intakes on seventh day of life, did not differ between the three study groups. However, there was an overall increase in total energy intake (i.v. + p.o.,) between seventh day of life and 10 $(72 \pm 19$ versus $83 \pm 21 \mathrm{kcal} / \mathrm{kg} / \mathrm{d}, p<0.001)$ that is accounted for by the expected advancement in enteral feeds.

Effect of exposure to oxygen on systemic inflammation. There was a positive correlation between the amount of oxygen infants were exposed to $\left(\mathrm{FiO}_{2}\right)$ and plasma cytokine levels $\left(r^{2}=0.28\right.$ for IL-6 and $r^{2}=0.31$ for IL-8, $n=50 ; p<$ $0.001)$. Overall, cytokine levels also correlated positively with $E\left(r^{2}=0.20\right.$ for IL-6; $r^{2}=0.20$ for IL-8, $\left.n=50 ; p<0.002\right)$;

Table 1. Clinical characteristics of infants

\begin{tabular}{|c|c|c|c|}
\hline Variable & AA $(n=9)$ & $\operatorname{LE}(n=9)$ & $\mathrm{LP}(n=8)$ \\
\hline $\mathrm{GA}$, mean $\pm \mathrm{SD}(\mathrm{wk})$ & $26 \pm 1$ & $26 \pm 1$ & $26 \pm 0$ \\
\hline Birth weight, mean $\pm \mathrm{SD}(\mathrm{g})$ & $751 \pm 211$ & $763 \pm 163$ & $749 \pm 118$ \\
\hline Gender, n $(\%)$ male & $5(63)$ & $6(60)$ & $3(38)$ \\
\hline Chorioamnionitis, n (\%) & $3(38)$ & $2(20)$ & $2(25)$ \\
\hline Apgar score at 5 min, mean $\pm \mathrm{SD}$ & $7 \pm 2$ & $6 \pm 2$ & $7 \pm 2$ \\
\hline SNAP score, median (IQ range) & $9(14-23)$ & $6(16-32)$ & $14(19-33)$ \\
\hline Culture-proven sepsis or NEC, $\mathrm{n}(\%)$ & $1(13)$ & $1(10)$ & $2(25)$ \\
\hline Glucose i.v. intake on day 7 , mean $\pm \mathrm{SD}(\mathrm{g} / \mathrm{kg} / \mathrm{d})$ & $10.3 \pm 2.0$ & $8.4 \pm 2.5$ & $10.0 \pm 2.2$ \\
\hline Protein i.v. intake on day 7 , mean $\pm \mathrm{SD}(\mathrm{g} / \mathrm{kg} / \mathrm{d})$ & $2.9 \pm 0.7$ & $2.5 \pm 0.8$ & $2.8 \pm 1.1$ \\
\hline Lipid i.v. intake on day 7 , mean $\pm \mathrm{SD}(\mathrm{g} / \mathrm{kg} / \mathrm{d})$ & $2.1 \pm 0.8$ & $2.6 \pm 0.8$ & $2.7 \pm 0.8$ \\
\hline Multivitamin i.v. intake on day 7, mean $\pm \mathrm{SD}(\mathrm{mL} / \mathrm{kg} / \mathrm{d})$ & $1.2 \pm 0.2$ & $1.1 \pm 0.3$ & $1.1 \pm 0.4$ \\
\hline Enteral intake on day 7, mean $\pm \mathrm{SD}(\mathrm{mL} / \mathrm{kg} / \mathrm{d})$ & $14 \pm 17$ & $20 \pm 22$ & $13 \pm 19$ \\
\hline Days of supplemental oxygen, median (IQ range) & $48(25-84)$ & $79(37-92)$ & $76(71-95)$ \\
\hline Fractional inspired oxygen, day 7 median (75th centile) & $0.23(0.30)$ & $0.23(0.25)$ & $0.24(0.26)$ \\
\hline Fractional inspired oxygen, day 10 median (75th centile) & $0.26(0.53)$ & $0.26(0.30)$ & $0.28(0.36)$ \\
\hline
\end{tabular}



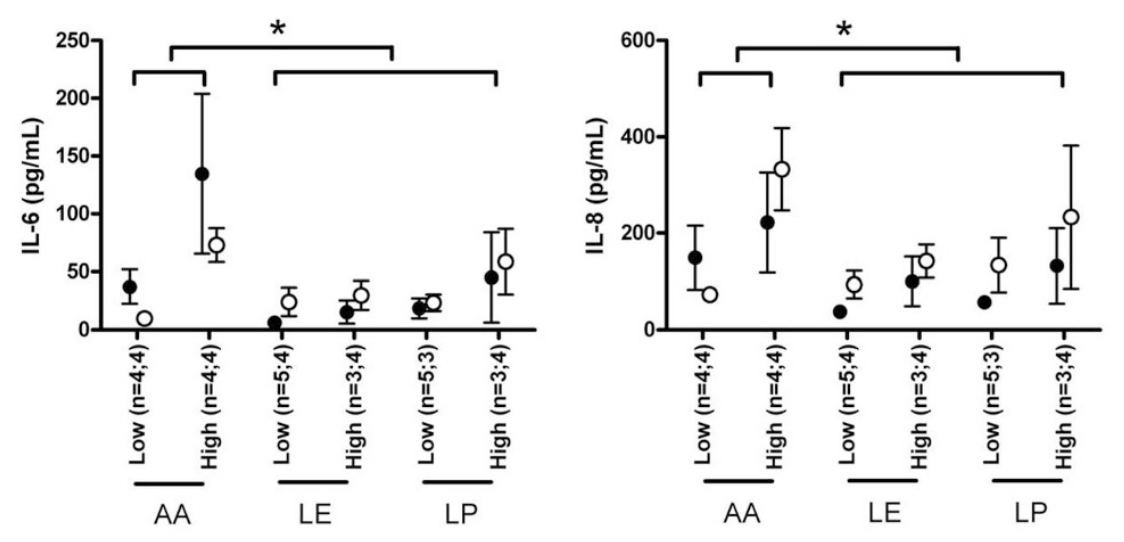

Figure 1. Plasma levels of IL-6 and IL-8 in preterm infants receiving TPN. Preterm infants were allocated to receive one of the following TPN regimens: AA, multivitamin (MVP) + amino acid/dextrose solution with lipid (LIP) provided separately $(n=9)$; LE, MVP administered with LIP exposed to light with amino acid/dextrose solutions provided separately $(n=9)$; LP, MVP administered with LIP protected from light with amino acid/dextrose solutions provided separately $(n=8)$. IL-6 (left panel) and IL-8 (right panel) were measured from plasma sampled on 7th day of life ( sample 1) and 10th day of life ( $\bigcirc$ sample 2). Data (mean $\pm \mathrm{SD}$ ) were compared by ANOVA between infants $(n=$ sample 1 ; sample 2) exposed to low $(<0.25)$ vs high $(\geq 0.25) \mathrm{FiO}_{2}$; ${ }^{*} p<0.05$. when analyzing samples 1 and 2 separately, this correlation reached statistical significance only for sample $2\left(r^{2}=0.40\right.$ for IL $6, n=24, p<0.001$ and $r^{2}=0.28$ for IL $8, n=24$, $p<0.01$ ). For IL- $1 \beta$, IL-10, IL-12, and TNF- $\alpha$, measurements were low and even below the level of detection in the majority of infants and therefore not shown.

Effect of TPN regimen on oxygen-associated systemic inflammation and oxidant stress. The analysis of variance showed an overall significant effect of oxygen on both IL-6 $\left(F_{1,34}=\right.$ $7.33, p<0.01)$ and IL-8 plasma concentrations $\left(F_{1,34}=9.92\right.$, $p<0.01$ ). Administration of MVPs with lipids seemed to protect against the oxygen-associated inflammatory response as a high $\mathrm{FiO}_{2}$ induced significantly lower IL-6 $\left(F_{1,34}=5.11\right.$, $p<0.05)$ and IL-8 $\left(F_{1,34}=4.48, p<0.05\right)$ cytokine responses in the LE and LP regimen, compared with the AA regimen (Fig. 1). Within our sample size, there was no additional detectable effect of light protection on the cytokine responses as no differences were found between LE and LP regimens $\left(F_{1,34}<1.6\right)$. Furthermore, no significant effect of duration of TPN [sample 1 versus sample $2,\left(F_{1,34}<4.0\right)$ ] was detected.

\section{DISCUSSION}

This study reports the effect of different modalities of coadministration of parenteral amino acid, lipid, and MVP solutions on the proinflammatory responses detected on exposure to supplemental oxygen. Infants in this study represent a subgroup of extremely low birth weight (ELBW) premature infants enrolled in a larger study designed to compare modalities of MVP admixture with TPN on antioxidant vitamin bioavailability and on the redox status of ELBW premature infants. In previous studies, we demonstrated that administering the parenteral LIP with multivitamins (whether light exposed or not) protected premature infants against the oxidant stress induced by oxygen supplementation $\left(\mathrm{FiO}_{2} \geq 25 \%\right)$, independently of antioxidant vitamin levels (Chessex P, unpublished data). A cutoff of $25 \% \mathrm{FiO}_{2}$ was chosen to better discriminate the clinical status of infants. In addition, oxidant stress has been reported with even relatively mild changes in supplemental oxygen (32). Furthermore, the inflammatory response to $\mathrm{FiO}_{2}>50 \%$ does not change substantially (33). We also confirmed that ELBW premature infants exhibited biologic markers of oxidant stress (redox potential of glutathione, di-tyrosine, and iso-PG- $F_{2 \alpha}$ ) that were significantly more oxidized than in adults (34), generally indicating a greater vulnerability to ROS-mediated tissue injury.

Similarly, in this subgroup, we demonstrate that the mode of administration of TPN modulates the inflammatory response induced by oxygen (Fig. 1). Indeed the AA in which MVP was mixed with the amino acid plus dextrose solution resulted in a greater systemic inflammatory response, as detected by levels of IL-6 and IL-8 in plasma. Both exposure to supplemental oxygen and TPN regimens influence markers of inflammation and oxidation. Modulation of the proinflammatory effect of oxygen by TPN can potentially be explained by a reduction in the generation of lipid and hydrogen peroxide, which likely impact on the activity of proinflammatory intracellular signaling events (e.g. NF-kB translocation to the nucleus) $(9,35)$. This model is supported by recent data showing that ROS, such as hydrogen peroxide, cause the scavenger protein thioredoxin (TRX) to dissociate from its thioredoxininteracting partner (TXNIP), which can then directly interact with the inflammasome component NLRP3 to cause production of an inflammatory responses (36). The infusion of TPN solutions containing peroxides, which are relatively stable and permeable oxidants, would also induce a traditional "insideout" stressor in the vessels, directly affecting the integrity of the endothelium (37). It has recently been proposed that stimulation of cytokines can also induce an "outside-in" paradigm that produces in turn a large amount of $\mathrm{H}_{2} \mathrm{O}_{2}$ in the vessel wall, potentiating an inflammatory response and causing initiation of necrosis, hypertrophy, and angiogenesis (35). This may account for some of the mechanisms (38) by which both inflammation and TPN are involved in the later development of bronchopulmonary dysplasia $(18,39)$. The presence of positive linear correlations between the redox potential and the systemic cytokine response supports a similar physiologic link between these two stressors in preterm infants.

Limitations of this study include the small sample size and the short duration of observation. However, the effect was reproducible at two time points (samples 1 and 2) even if both samples were not systematically distributed in the same high or low oxygen groups. The ANOVA showed no statistically significant effect related to samples. Although the clinical 
characteristics of infants in the three groups are not statistically different, a logistic regression model to exclude other confounders was not performed because of the limited sample size. The clinical complications of inflammation could have extended well beyond the treatment period of our protocol, which was limited to the first 2 wk of life.

In conclusion, supplemental oxygen exposure is associated with a systemic inflammatory response, detected by measuring plasma levels of IL-6 and IL-8: two significant markers of adverse long-term pulmonary and neurodevelopmental outcomes in preterm infants. Delivering light-exposed lipids separate from amino acids may enhance the proinflammatory effect of oxygen. Furthermore, we provide compelling evidence that the proinflammatory response generated by supplemental oxygen exposure is attenuated in infants exposed to lipids mixed with the multivitamin preparation. Therefore, the different modalities of coadministration of TPN components may have important protective effects against potentially deleterious inflammation. These results need to be confirmed on a larger sample size incorporating relevant clinical outcomes.

Acknowledgments. We thank Indira Genowati and Mihoko Whalen for experimental assistance with the cytokine measurements and Jas Aulack for technical assistance.

\section{REFERENCES}

1. Bohrer B, Silveira RC, Neto EC, Procianoy RS 2010 Mechanical ventilation of newborns infant changes in plasma pro- and anti-inflammatory cytokines. J Pediatr 156:16-19

2. Laborie S, Lavoie JC, Chessex P 2000 Increased urinary peroxides in newborn infants receiving parenteral nutrition exposed to light. J Pediatr 136:628-632

3. Fujinaga H, Baker CD, Ryan SL, Markham NE, Seedorf GJ, Balasubramanian V, Abman SH 2009 Hyperoxia disrupts vascular endothelial growth factor-nitric oxide signaling and decreases growth of endothelial colony-forming cells from preterm infants. Am J Physiol Lung Cell Mol Physiol 297:L1160-L1169

4. Rozycki HJ, Comber PG, Huff TF 2002 Cytokines and oxygen radicals after hyperoxia in preterm and term alveolar macrophages. Am J Physiol Lung Cell Mol Physiol 282:L1222-L1228

5. Yeung MY 2006 Influence of early postnatal nutritional management on oxidative stress and antioxidant defence in extreme prematurity. Acta Paediatr 95:153-163

6. Warner BB, Wispe JR 1992 Free radical-mediated diseases in pediatrics. Semin Perinatol 16:47-57

7. Vento M, Moro M, Escrig R, Arruza L, Villar G, Izquierdo I, Roberts LJ 2nd, Arduini A, Escobar JJ, Sastre J, Asensi MA 2009 Preterm resuscitation with low oxygen causes less oxidative Stress, inflammation, and chronic lung disease. Pediatrics 124:439-449

8. Imai Y, Kuba K, Neely GG, Yaghubian-Malhami R, Perkmann T, van Loo G, Ermolaeva M, Veldhuizen R, Leung YH, Wang H, Liu H, Sun Y, Pasparakis M, Kopf M, Mech C, Bavari S, Peiris JS, Slutsky AS, Akira S, Hultqvist M, Holmdahl R, Nicholls J, Jiang C, Binder CJ, Penninger JM 2008 Identification of oxidative stress and toll-like receptor 4 signaling as a key pathway of acute lung injury. Cell 133:235-249

9. de Oliveira-Marques V, Cyrne L, Marinho HS, Antunes F 2007 A quantitative study of NF-kappaB activation by $\mathrm{H} 2 \mathrm{O} 2$ : relevance in inflammation and synergy with TNF-alpha. J Immunol 178:3893-3902

10. Volpe JJ 2008 Postnatal sepsis, necrotizing enterocolitis, and the critical role of systemic inflammation in white matter injury in premature infants. J Pediatr 153:160-163

11. Yusa T, Beckman JS, Crapo JD, Freeman BA 1987 Hyperoxia increases H2O2 production by brain in vivo. J Appl Physiol 63:353-358
12. Gerstner B, Buhrer C, Rheinlander C, Polley O, Schuller A, Berns M, Obladen M, Felderhoff-Mueser U 2006 Maturation-dependent oligodendrocyte apoptosis caused by hyperoxia. J Neurosci Res 84:306-315

13. Normann E, Lacaze-Masmonteil T, Eaton F, Schwendimann L, Gressens P, Thebaud B 2009 A novel mouse model of Ureaplasma-induced perinatal inflammation: effects on lung and brain injury. Pediatr Res 65:430-436

14. Gerstner B, DeSilva TM, Genz K, Armstrong A, Brehmer F, Neve RL, FelderhoffMueser U, Volpe JJ, Rosenberg PA 2008 Hyperoxia causes maturation-dependent cell death in the developing white matter. J Neurosci 28:1236-1245

15. Viscardi RM, Muhumuza CK, Rodriguez A, Fairchild KD, Sun CC, Gross GW, Campbell AB, Wilson PD, Hester L, Hasday JD 2004 Inflammatory markers in intrauterine and fetal blood and cerebrospinal fluid compartments are associated with adverse pulmonary and neurologic outcomes in preterm infants. Pediatr Res 55:1009-1017

16. Yoon BH, Romero R, Jun JK, Park KH, Park JD, Ghezzi F, Kim BI 1997 Amniotic fluid cytokines (interleukin-6, tumor necrosis factor-alpha, interleukin-1 beta, and interleukin-8) and the risk for the development of bronchopulmonary dysplasia. Am J Obstet Gynecol 177:825-830

17. Ambalavanan N, Carlo WA, D'Angio CT, McDonald SA, Das A, Schendel D, Thorsen P, Higgins RD 2009 Cytokines associated with bronchopulmonary dysplasia or death in extremely low birth weight infants. Pediatrics 123:1132-1141

18. Paananen R, Husa AK, Vuolteenaho R, Herva R, Kaukola T, Hallman M 2009 Blood cytokines during the perinatal period in very preterm infants: relationship of inflammatory response and bronchopulmonary dysplasia. J Pediatr 154:39-43, e3

19. Ehrenkranz RA, Walsh MC, Vohr BR, Jobe AH, Wright LL, Fanaroff AA, Wrage LA, Poole K 2005 Validation of the National Institutes of Health consensus definition of bronchopulmonary dysplasia. Pediatrics 116:1353-1360

20. Lucas A, Morley R, Cole TJ 1998 Randomized trial of early diet in preterm babies and later intelligence quotient. BMJ 317:1481-1487

21. Silvers KM, Sluis KB, Darlow BA, McGill F, Stocker R, Winterbourn CC 2001 Limiting light-induced lipid peroxidation and vitamin loss in infant parenteral nutrition by adding multivitamin preparations to Intralipid. Acta Paediatr 90:242-249

22. Lavoie JC, Bélanger S, Spalinger M, Chessex P 1997 Admixture of a multivitamin preparation to parenteral nutrition: the major contributor to in vitro generation of peroxides. Pediatrics 99:E6

23. Neuzil J, Darlow BA, Inder TE, Sluis KB, Winterbourn CC, Stocker R 1995 Oxidation of parenteral lipid emulsion by ambient and phototherapy lights: potential toxicity of routine parenteral feeding. J Pediatr 126:785-790

24. Helbock HJ, Motchnik PA, Ames BN 1993 Toxic hydroperoxides in intravenous lipid emulsions used in preterm infants. Pediatrics 91:83-87

25. Knafo L, Chessex P, Rouleau T, Lavoie JC 2005 Association between hydrogen peroxide-dependent byproducts of ascorbic acid and increased hepatic acetyl-CoA carboxylase activity. Clin Chem 51:1462-1471

26. Laborie S, Lavoie JC, Pineault M, Chessex P 1999 Protecting solutions of parenteral nutrition from peroxidation. JPEN J Parenter Enteral Nutr 23:104-108

27. Khashu M, Harrison A, Lalari V, Gow A, Lavoie JC, Chessex P 2006 Photoprotection of parenteral nutrition enhances advancement of minimal enteral nutrition in preterm infants. Semin Perinatol 30:138-145

28. Redline RW, Faye-Petersen O, Heller D, Qureshi F, Savell V, Vogler C 2003 Amniotic infection syndrome: nosology and reproducibility of placental reaction patterns. Pediatr Dev Pathol 6:435-448

29. Richardson DK, Corcoran JD, Escobar GJ, Lee SK 2001 SNAP-II and SNAPPE-II: simplified newborn illness severity and mortality risk scores. J Pediatr 138:92-100

30. Lavoie JC, Rouleau T, Tsopmo A, Friel J, Chessex P 2008 Influence of lung oxidant and antioxidant status on alveolarization: role of light-exposed total parenteral nutrition. Free Radic Biol Med 45:572-577

31. Schafer FQ, Buetner GR 2001 Redox environment of the cell as viewed through the redox state of the glutathione disulfide/glutathione couple. Free Radic Biol Med 30:1191-1212

32. Smith CV, Hansen TN, Martin NE, McMicken HW, Elliot SJ 1993 Oxidant stress response in premature infants during exposure to hyperoxia. Pediatr Res 34:360-365

33. Harling AE, Beresford MW, Vince GS, Yoxall CW 2005 Does the use of $50 \%$ oxygen at birth in preterm infants reduce lung injury? Arch Dis Child Fetal Neonatal Ed 90:F401-F405

34. Belik J, Gonzalez-Lius GE, Perez-Vizcaino F, Villamor E 2010 Isoprostanes in fetal and neonatal health and diseases. Free Radic Biol Med 48:177-188

35. Brown DI, Griendling KK 2009 Nox proteins in signal transduction. Free Radic Biol Med 47:1239-1253

36. Zhou R, Tardivel A, Thorens B, Choi I, Tschopp J 2010 Thioredoxin-interacting protein links oxidative stress to inflammasome activation. Nat Immunol 11:136-140

37. Lavoie JC, Chessex P 1994 Gender-related response to a tert-butyl hydroperoxideinduced oxidation in human neonatal tissue. Free Radic Biol Med 16:307-313

38. Jobe AJ 1999 The new BPD: an arrest of lung development. Pediatr Res 46:641-643

39. Bassiouny MR, Almarsafawy H, Abdel-Hady H, Nasef N, Hammad TA, Aly H 2009 A randomized controlled trial on parenteral nutrition, oxidative stress, and chronic lung disease in preterm infants. J Pediatr Gastroenterol Nutr 48:363-369 